\title{
Outcomes of aggressive treatment in esophageal cancer patients with synchronous solitary brain metastasis
}

\author{
CEM ONAL, BERNA AKKUS YILDIRIM and OZAN CEM GULER \\ Department of Radiation Oncology, Baskent University Faculty of Medicine, Adana \\ Dr Turgut Noyan Research and Treatment Centre, 01120 Adana, Turkey
}

Received November 1, 2016; Accepted March 3, 2017

DOI: $10.3892 / \operatorname{mco} .2017 .1263$

\begin{abstract}
The aim of the present study was to investigate the outcomes of esophageal cancer (EC) patients with isolated synchronous brain oligometastasis (oligo-BM) treated with chemoradiotherapy (CRT) of the primary site and localized treatment of the BM with surgery, radiotherapy (RT) or radiosurgery. Of 125 EC patients investigated, seven patients (6\%) had solitary BM. Six patients were diagnosed prior to, and one patient was diagnosed during, treatment. All patients were treated with neoadjuvant chemotherapy and whole-brain RT (WBRT) for BM. All but one patient received definitive CRT with a median RT dose of 50.4 Gy using conventional fractionation RT. The median age at diagnosis was 59 years (range, 48-77 years). Six patients succumbed to mortality, and one continued to receive systemic chemotherapy at the last visit. The median survival time of the patients was 18.9 months (range, 10.0-27.2 months). Median time to progression after completion of the treatments was 8 months (range, 3-9 months). Two patients had progression of the primary tumor, and one patient had progression of the BM. The neurological status of three patients with BM who were identified during the staging work-up did not deteriorate as a consequence of WBRT. In conclusion, the present study has demonstrated that aggressive treatment of the primary tumor and oligo-BM in patients with EC may prolong the survival time.
\end{abstract}

\section{Introduction}

Esophageal cancer (EC) is one of the most common tumors of the gastrointestinal system. The prognosis of EC patients is relatively poor, since the majority of patients are diagnosed at an advanced stage. The most common metastatic sites

Correspondence to: Professor Cem Onal, Department of Radiation Oncology, Baskent University Faculty of Medicine, Adana Dr Turgut Noyan Research and Treatment Centre, 01120 Adana, Turkey E-mail: hcemonal@hotmail.com

Key words: esophageal cancer, brain metastasis, oligometastasis, radiotherapy, chemoradiotherapy include the lungs, liver, and bones (1). The incidence of brain oligometastasis (oligo-BM) is extremely rare (1-3\% incidence in clinical series) $(2,3)$. The majority of patients with BM are diagnosed at an advanced clinical stage, and the majority of BMs tend to occur together with other organ metastases $(2,4,5)$.

Since brain imaging as part of a metastasis work-up is not routinely performed, detection of solitary BM at diagnosis is difficult. However, with the increased use of positron emission tomography (PET) at initial staging, along with advances in neuroimaging, the incidence of BM has gradually increased, as reported in the literature $(6,7)$. Due to the rarity of BM in patients with EC, there is no standardized treatment for these patients. The survival time of patients with EC and metastasis to the brain ranges from 2 weeks to 25 months, depending on the extent of disease and the treatment modalities employed $(7,8)$. Previously, it was demonstrated that patients with one to three BMs had improved survival rates with advanced treatment modalities (9). Therefore, an aggressive treatment approach may be effective for only a limited number of patients with good performance status and solitary metastasis. However, it is important to make balanced clinical decisions for treatment planning; specifically, how to identify patients with oligometastasis who would benefit from localized therapy. How long localized therapy may be able to extend life expectancy has yet to be determined for EC patients with BM. In the present study, the long-term survival of seven EC patients with isolated synchronous BM treated with definitive chemoradiotherapy (CRT) of the primary site, and localized treatments such as surgery, radiotherapy (RT) or radiosurgery of the BM, is reported.

\section{Patients and methods}

Patient population. A retrospective study of the clinical records from 125 patients with EC who had been treated at Baskent University Faculty of Medicine Department of Radiation Oncology, Adana, Turkey, between October 2007 and December 2015 was designed. Of these 125 patients, 10 patients $(8 \%)$ had BM. Seven of the 10 patients $(6 \%)$ had solitary BM diagnosed prior to or during treatment, while three patients $(2 \%)$ had multiple BMs (two patients had multiple BMs only, and one patient had BM and lung metastasis). The seven patients with a solitary BM were chosen for analysis, and their characteristics are summarized in Table I. In these patients, 
Table I. Characteristics of EC patients with solitary BM.

\begin{tabular}{|c|c|c|c|c|c|c|c|}
\hline $\begin{array}{l}\text { Patient } \\
\text { no. }\end{array}$ & Age, sex & Diagnosis & Stage & Location & $\begin{array}{l}\text { Treatment } \\
\text { (esophagus) }\end{array}$ & $\begin{array}{l}\text { Treatment } \\
\text { (BM) }\end{array}$ & $\begin{array}{l}\text { Systemic } \\
\text { treatment }\end{array}$ \\
\hline 1 & $50, \mathrm{M}$ & Adeno $\mathrm{Ca}$ & T4N1 & Distal 1/3 & CRT (54 Gy + capecitabine) & WBRT & $\begin{array}{c}6 \times \text { cisplatin } \\
+ \text { capecitabine }\end{array}$ \\
\hline 2 & $48, \mathrm{M}$ & Adeno $\mathrm{Ca}$ & T3N1 & Distal $1 / 3$ & CRT (54 Gy + 5-FU) & Surgery + WBRT & $6 \times \mathrm{CFF}$ \\
\hline 3 & $48, \mathrm{M}$ & Adeno $\mathrm{Ca}$ & T3N1 & Distal $1 / 3$ & CRT (60 Gy+ 5-FU) & GK + WBRT & $6 \times \mathrm{ECF}$ \\
\hline 4 & $76, \mathrm{M}$ & Adeno $\mathrm{Ca}$ & T4N0 & Distal 1/3 & CRT (50.4 Gy + 5-FU) & Surgery + WBRT & $6 \times$ FU-FA \\
\hline 5 & $59, \mathrm{M}$ & SCC & T4N1 & Mid 1/3 & CRT (60 Gy + cisplatin) & Surgery + WBRT & $6 \times \mathrm{CFF}$ \\
\hline 6 & $77, \mathrm{M}$ & Adeno $\mathrm{Ca}$ & T3N1 & Distal 1/3 & CRT (54 Gy + capecitabine) & WBRT & $8 \times$ capecitabine \\
\hline 7 & $69, \mathrm{M}$ & SCC & T3NO & Proximal 1/3 & $\mathrm{RT}(60 \mathrm{~Gy})$ & WBRT & $6 \times \mathrm{CFF}$ \\
\hline
\end{tabular}

EC, esophageal cancer; BM, brain metastasis; M, male; Adeno Ca, adenocarcinoma; SCC, squamous cell carcinoma; CRT, chemoradiotherapy; 5-FU, 5-fluorouracil; RT, radiotherapy; WBRT, whole-brain radiotherapy; GK, Gamma Knife; CFF, cisplatin/5-fluorouracil/folinic acid, ECF, epirubicin/cisplatin/5-fluorouracil; FA, folinic acid.

BM was diagnosed via PET, or a combination with computed tomography (CT) and magnetic resonance imaging (MRI). In addition, three patients received histological confirmation of the condition following surgical resection of the brain lesion. Written informed consent was obtained from each patient prior to inclusion of their data in the present study.

Treatment of primary tumors. Aggressive treatment of the primary tumors was performed in three stages. First, seven patients with solitary BM were treated with neoadjuvant chemotherapy for treatment of the primary tumors (Table I). One patient was diagnosed with BM following the second cycle of chemotherapy. Three patients received six cycles of cisplatin and 5-fluorouracil (5-FU); one patient received six cycles of epirubicin, cisplatin, and 5-FU; one patient received six cycles of cisplatin and capecitabine; one patient received six cycles of 5-FU and folinic acid; and one patient received eight cycles of capecitabine. Following completion of the neoadjuvant chemotherapy, PET-CT was used for restaging and assessing the treatment response (Fig. 1). The primary tumor was treated in cases with no progression of the disease following systemic chemotherapy. In the second course of treatment, all the patients received definitive CRT with a median RT dose of 50.4 Gy (range, 50.4-60 Gy) using conventional fractionation with either a 3-dimensional conformal (two patients) or intensity-modulated (five patients) RT technique. One patient refused additional treatment, and received palliative care only. Finally, six of the seven patients underwent a second round of chemotherapy: Three patients received 5-FU, two patients received capecitabine, and one received cisplatin concurrently with RT. All the patients tolerated the treatment well; no serious side-effects were observed during or after completion of the treatment.

Patients with multiple BMs were treated with palliative RT delivered to the primary tumor site after whole-brain RT (WBRT), followed by systemic chemotherapy.

Treatment of BMs. Six of seven patients had solitary BM diagnosed prior to initiation of any treatment, whereas in one patient, $\mathrm{BM}$ was identified at the end of the second cycle of chemo- therapy. Four patients reported headache and nausea/vomiting at the first diagnosis, and BM was confirmed using MRI. In three patients, no neurological symptoms were observed, and BM was identified incidentally by initial PET-CT (Fig. 2). Although the treatment regimens of EC patients with BM were determined by the treating oncologists, all the patients underwent WBRT. Three patients underwent surgical resection followed by WBRT, and one patient also had Gamma Knife radiosurgery prior to WBRT. WBRT was administered via a $6 \mathrm{MV}$ linear accelerator in daily fractions of $3 \mathrm{~Gy}$ for a total dose of 30 Gy delivered in 10 fractions. The Gamma Knife radiosurgery dose was $18 \mathrm{~Gy}$, delivered to the solitary metastasis. All the patients tolerated the treatment well; only corticosteroids were administered during the RT, if required.

\section{Results}

Patient outcomes for patients with solitary BM. The median age for the seven patients at diagnosis was 59 years (range, 48-77 years). All the patients were male, and six succumbed to mortality resulting from their cancer (Table II). The median survival time of the patients was 18.9 months (range, 10.0-27.2 months). The median time to progression after completion of all the treatments was 8 months (range, 3-9 months). Two patients had tumor progression at the primary site, and one patient had BM progression. Two patients with localized progression of the primary tumor also had intra-abdominal lymph node metastasis, which was observed 3 and 9 months, respectively, following completion of the treatment. These patients received systemic chemotherapy: One patient succumbed to the cancer as a consequence of disease progression, whereas the other patient remained alive, although with the disease (Table II). The patient who had progression of the BM was treated with excision of the BM, followed by WBRT. Eight months following completion of the treatment, new metastatic foci in the brain and lung were identified (Fig. 3). Evidence of systemic progression was observed at multiple sites, although mostly in the liver and lungs. Two patients with adenocarcinoma had liver metastasis only at 5 and 9 months, respectively, after the completion of treatment. 

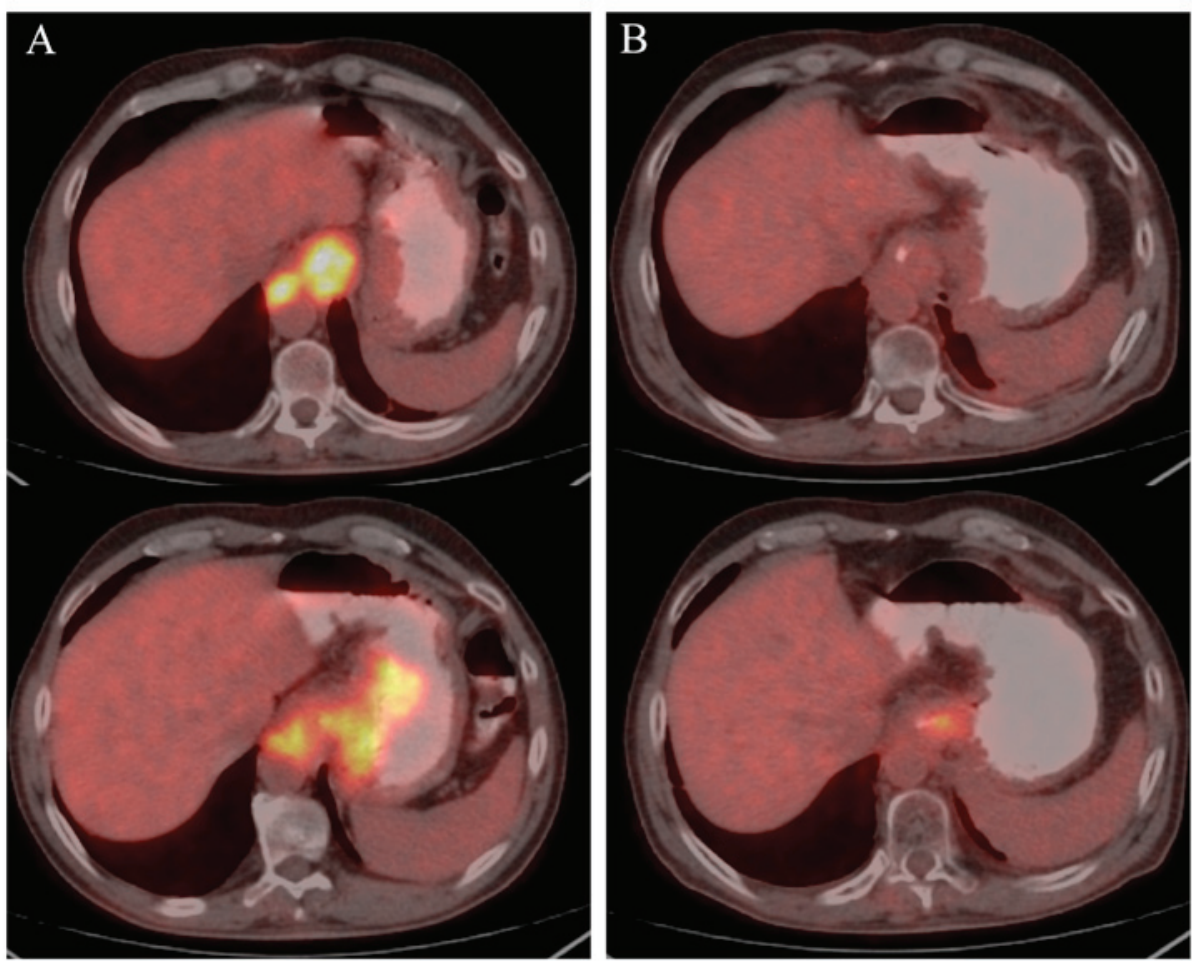

Figure 1. PET-CT was used for restaging and assessing the treatment response after completion of neoadjuvant chemotherapy. FDG-PET/CT images of patient no. 4 are shown. (A) Increased FDG uptake at the distal esophagus and gastroesophageal junction [maximum standardized uptake value $\left(\mathrm{SUV}_{\max }\right)=15.6$ prior to initiation of the treatment]. (B) Marked response observed following six cycles of 5-fluorouracil and folinic acid treatment. PET, positron emission tomography; CT, computed tomography; FDG, ${ }^{18} \mathrm{~F}$-fluorodeoxyglucose.
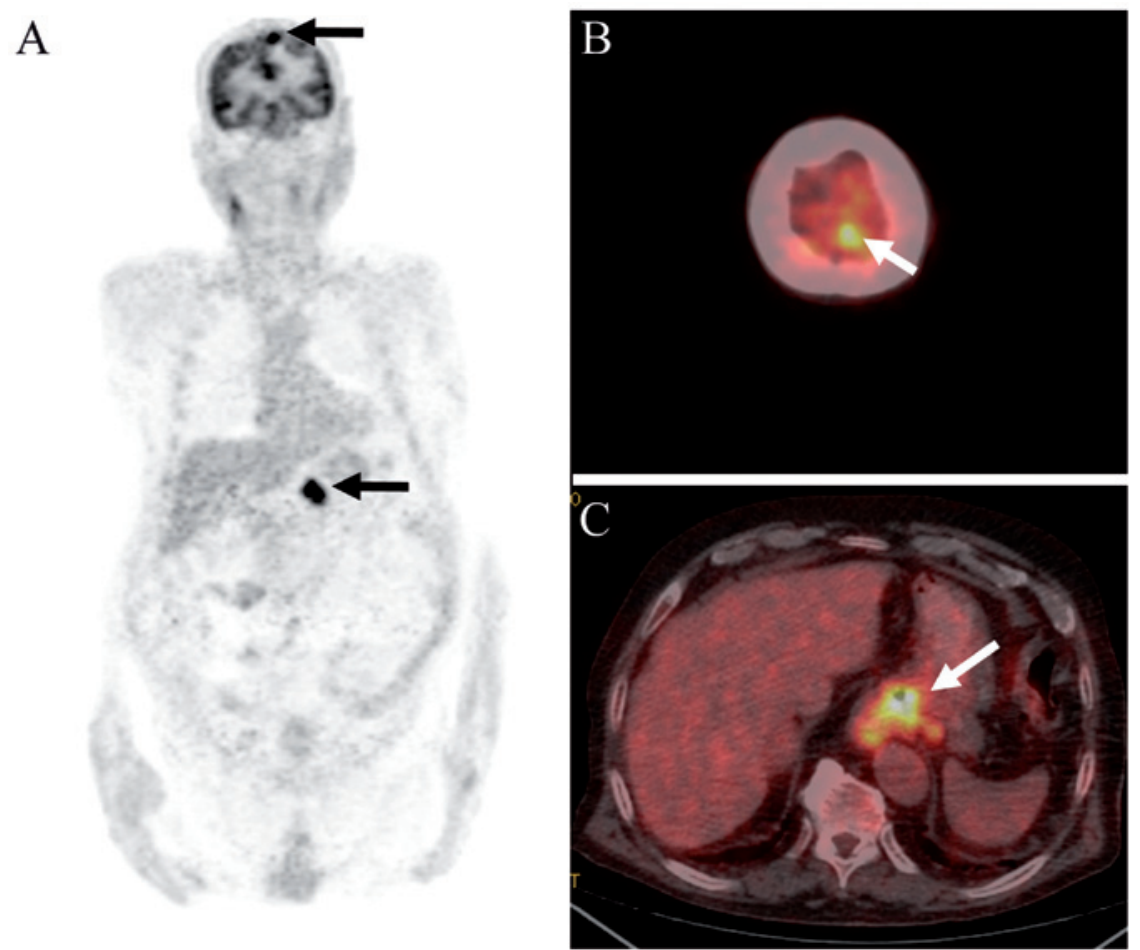

Figure 2. A representative patient, where BM was identified incidentally by initial PET-CT. FDG-PET/CT images of patient no. 6. (A) FDG-PET image, demonstrating increased uptake in the brain (arrow), and distal esophagus (arrow) indicating the presence of the primary tumor and BM. (B and C) Overlaid PET and CT images, demonstrating increased FDG uptake in the the parietal region of the brain [maximum standardized uptake value $\left.\left(\mathrm{SUV}_{\max }\right)=11.6\right]$ and distal esophagus $\left(\mathrm{SUV}_{\max }=12.4\right)$. BM, brain metastasis; PET, positron emission tomography; CT, computed tomography; FDG, ${ }^{18} \mathrm{~F}$-fluorodeoxyglucose.

One patient had liver metastasis, together with lung metastasis and BM 9 months after completion of the treatment. One patient refused systemic chemotherapy, and succumbed from the disease under supportive care. 
Table II. Treatment outcomes for EC patients with BM.

\begin{tabular}{lcccc} 
Patient no. & Progression site & Status & $\begin{array}{c}\text { Time to progression } \\
\text { (months) }\end{array}$ & $\begin{array}{c}\text { Overall survival time } \\
\text { (months) }\end{array}$ \\
\hline 1 & Esophagus, intra-abdominal ln & DwD & 3 & 10.0 \\
2 & Lung, brain & DwD & 8 & 20.3 \\
3 & Liver & DwD & 5 & 20.6 \\
4 & Liver & DwD & 9 & 27.2 \\
5 & Lung & DwD & 5 & 13.9 \\
6 & Esophagus, intra-abdominal ln & AwD & 9 & 14.5 \\
7 & Lung, liver, bones & DwD & 9 & 18.9 \\
\hline
\end{tabular}

EC, esophageal cancer; BM, brain metastasis; ln, lymph node; DwD, died with disease; AwD, alive with the disease.

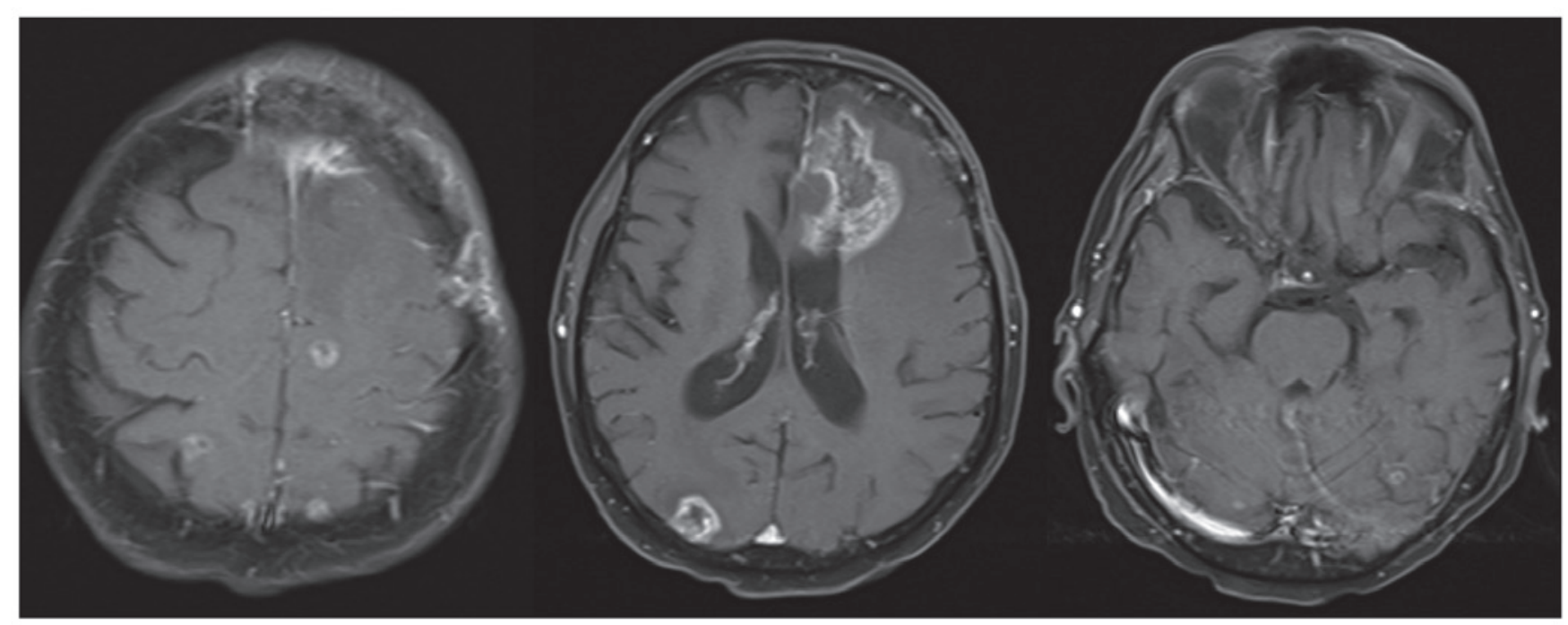

Figure 3. Magnetic resonance images of patient no. 2, demonstrating new metastatic foci and progression of primary metastatic lesions, observed 8 months after completion of all the treatments.

Neurological symptoms at diagnosis in four patients were resolved completely after the conclusion of WBRT. Mild to moderate headache, which was resolved following corticosteroid administration, was observed during RT. The neurological status of three patients with BM that had been identified incidentally during staging work-up did not deteriorate after WBRT.

Outcomes for patients with multiple BMs. Two patients with multiple BMs, who were provided palliative treatment, only survived 2.1 and 3.1 months after the diagnosis. One of these patients only had BM, whereas the other patient had liver metastasis together with BM.

Six patients were diagnosed with multiple BMs at a median of 2.5 months (range 0.8-16.2 months) following completion of the primary tumor treatment. Three of six patients had BM only, while one patient had local recurrence, one patient had liver metastasis, and one patient had lung and liver metastasis, together with multiple BMs. The median survival of patients with BM observed after completion of the curative treatment of EC was 5.0 months (range, 1.0-9.6 months).

\section{Discussion}

In the present patient series, the efficacy of treatment of both $\mathrm{BM}$ and primary tumor in EC patients with synchronous solitary BM was investigated, and longer survival times were observed.

BM from EC is extremely rare, and only limited information is available. In the literature, the incidence of BM was $0-2 \%$ in clinical studies, and $0-5.1 \%$ in autopsy studies $(3,4)$. Kanemoto et al (10) reported that the crude and 3-year cumulative incidences of BM in 391 patients with EC following CRT were 3.1 and $6.6 \%$, respectively. However, in the majority of studies, the incidence of BM has probably been underestimated since, in most series, routine brain imaging is not typically part of the metastatic work-up. In the current study, the incidence of $\mathrm{BM}$ was $6 \%$, which was slightly higher compared with previously published series (3-6). Although patients with BM were not routinely evaluated with MRI in the present study, in four patients with neurological symptoms, cranial CT or MRI was performed to rule out BM. Furthermore, the majority of patients with $\mathrm{EC}$ were evaluated with ${ }^{18} \mathrm{~F}$-fluorodeoxyglucose (FDG)-PET/CT for initial staging and RT planning. In three 
patients, the existence of BM was demonstrated incidentally with FDG-PET/CT, although these patients did not have any neurological symptoms. Since it was reported that the sensitivity of PET-CT for detecting BM in other cancer types was $70 \%$, and the specificity was very high (11), the higher incidence of BM in the current study may be connected with higher rates of PET-CT use compared with previous studies.

There have been several efforts to assess whether neuro staging is essential for patients with EC. Gabrielsen et al (8) explored the usefulness of preoperative cranial CT to investigate occult metastases in a cohort of 240 patients who underwent esophagectomy. However, none of the patients were found to have occult metastases by CT. The authors suggested that a consistent, true incidence of BM from EC in their material could not be determined exactly by $\mathrm{CT}$, and that the crude incidence of $\mathrm{BM}$ was $3.6 \%$ if MRI were to have been used instead of CT. Studies detecting BM in routine staging are very rare; the majority of studies involved lung cancer patients $(12,13)$. In a study by Seute et al (12), the incidence of BM in 481 patients with small cell lung cancer was $10 \%$ with CT, but increased up to $24 \%$ with MRI. Furthermore, in the CT group, all the patients diagnosed with BM were symptomatic, whereas in the MRI group, $11 \%$ of the patients were symptomatic. These authors concluded that the estimated incidence of BM increased when MRI was used instead of CT. Hjorthaug et al (13) assessed whether PET-CT was suitable for selecting patients for MRI on suspicion of BM in 596 patients with lung cancer. The sensitivity, specificity, and positive predictive values were 72,100 , and $97 \%$, respectively, and the authors concluded that PET-CT may be suitable for selecting patients for MRI in diagnostic centers that did not perform routine MRI in the pre-therapeutic staging work-up. The results of the present study also supported this finding: Higher rates of oligo-BM were detected at the time of EC diagnosis, which could have been due to the routine use of FDG-PET/CT for initial staging for the majority of the cases.

The stage of the primary tumor is the most important risk factor for survival of patients with EC (2). Similarly, BM tended to occur in patients with advanced clinical disease (4), which was supported by the present series. In the current study, all the patients had clinical-stage T3 or T4 disease, and five of seven patients also had clinical lymph node metastasis. Another risk factor for predicting BM in patients with EC is mean tumor length. It was previously reported that primary tumor length in EC patients without BM was shorter compared with EC patients with $\mathrm{BM}(4,8)$. In addition, treatment modality influences the time to BM development. Song et al (4) demonstrated that the median time to BM development was longer in patients treated with CRT compared with patients treated with chemotherapy alone (15.2 vs. 7.6 months). These findings indicated that patients with an extensive clinical stage have a high risk for developing BM, and treatment of the primary tumor with definitive CRT may extend the time prior to BM occurrence. In the current study, another factor contributing towards longer survival time was connected with successful local treatment with definitive CRT. Only two patients had local recurrence, together with intra-abdominal lymph node metastasis.

The prognosis is poor for EC patients with BM, and only limited data are available concerning treatment approaches.
The median survival time after diagnosis of BM was only 3.8 months, according to a report by Weinberg et al (5). However, it was reported in certain series that surgical resection of isolated BM in EC patients, with or without WBRT, resulted in a 3.8-26.2 month median survival time $(2,5,14)$. In the largest series reported by Ogawa et al (2), the mean survival time was 1.8 months in patients treated with WBRT alone, 3.8 months in patients who underwent surgical resection of a solitary BM, and 9.6 months in patients with EC treated with WBRT following surgical resection of a solitary BM. However, 5 of 36 patients who underwent postoperative WBRT, and who had no active extracranial disease, survived $>1$ year. Weinberg et al (5) demonstrated in a study of 27 patients that the longest survival time was observed in patients with single BM who underwent surgery and WBRT (median survival, 9.6 months), WBRT alone, surgery, surgery and WBRT, or stereotactic radiosurgery (SRS) alone. Yoshida (14) evaluated the treatment outcomes of patients with BM treated with WBRT alone, surgery alone, and postoperative WBRT. The median survival time of patients with single BM treated with SRS was 38.2 months, compared with 16.4 months for patients with multiple BMs. The author concluded that the best outcome was observed in patients treated with surgical resection followed by RT. Song et al (4) reported a median survival time of 4.2 months for 26 patients with BM (12 with single BM, and 14 with multiple BMs); the longest survival time was 7 months in patients who underwent surgery and postoperative RT. In the present series, although patients treated with postoperative WBRT or SRS and WBRT survived slightly longer compared with patients treated with WBRT alone, the progression of BM was observed only in one patient treated with postoperative WBRT. However, patients with multiple BMs at diagnosis who were provided palliative treatment only had very low survival rates, which may have been due to disease dissemination.

The kinetics of development of micrometastases, and particularly of oligometastases, was explored after having made certain assumptions. It is accepted that, beyond a threshold for the initiation of metastatic spread, which varies widely among different tumor types, the rate of primary tumor deposits with metastatic potential increases exponentially (15). Therefore, the delivery of metastatic clonogens from the primary tumor is accompanied by a similar exponential growth of each micrometastasis that becomes newly established at another site. The aim of treatment of oligometastatic disease has the potential to prevent further evolution of genetically unstable clones and metastatic spread, which may potentially improve overall disease control $(16,17)$. Multiple trials are under way to determine whether localized treatment of oligometastatic disease has been beneficial in various types of cancers; however, no data on EC patients with BM are available in the literature.

The present study had several limitations. First, this was a retrospective study. Secondly, the patient number was relatively limited, and therefore it was difficult to draw strong conclusions. Thirdly, there may have been patient selection bias: Although patients with single BM were analyzed who had an improved performance status, in the current study, patients with multiple BMs had a worse performance status and disseminated disease. Therefore, it was not possible perform a head-to-head comparison. Patients with multiple BMs who exhibited a good treatment response should also be 
evaluated. Finally, patients who received different treatment regimens were included in the analysis, which might have affected treatment outcomes. In the present series, cases were selected according to an approximately similar treatment strategy, which was neoadjuvant chemotherapy with treatment of BM at diagnosis, followed by definitive CRT or RT of the primary tumor site, even in cases where a good treatment response was observed.

Nevertheless, the present study has produced a number of important findings. Improved survival times were observed in selected patients treated with aggressive systemic and local treatment modalities. In addition, patients with oligometastasis are an important population, and the survival time of this population may be extended with appropriate diagnostic and treatment modalities.

In conclusion, the present study is, to the best of our knowledge, the first to demonstrate the efficacy of aggressive local and systemic treatment of the primary tumor site and BM of patients with EC. Although the prognosis of EC with BM is relatively poor, the findings in this study have revealed that the long-term survival of EC patients with BM may be augmented by locally ablative treatment of the primary tumor and the BM. Therefore, in selected patients, a curative approach with effective concurrent CRT, rather than palliative treatment, may result in improved local control and prolonged survival rates. With novel targeted therapeutics and effective adjuvant systemic therapies to control systemic disease, longer survival rates may be observed in selected EC patients with BM. Finally, physicians should be aware of the metastatic status of such patients prior to their deciding upon a treatment plan.

\section{References}

1. Quint LE, Hepburn LM, Francis IR, Whyte RI and Orringer MB Incidence and distribution of distant metastases from newly diagnosed esophageal carcinoma. Cancer 76: 1120-1125, 1995.

2. Ogawa K, Toita T, Sueyama H, Fuwa N, Kakinohana Y, Kamata M, Adachi G, Saito A, Yoshii Y and Murayama S: Brain metastases from esophageal carcinoma: natural history, prognostic factors, and outcome. Cancer 94: 759-764, 2002.

3. Go PH, Klaassen Z, Meadows MC and Chamberlain RS: Gastrointestinal cancer and brain metastasis: a rare and ominous sign. Cancer 117: 3630-3640, 2011.
4. Song Z, Lin B, Shao L and Zhang Y: Brain metastases from esophageal cancer: clinical review of 26 cases. World Neurosurg 81: 131-135, 2014.

5. Weinberg JS, Suki D, Hanbali F, Cohen ZR, Lenzi R and Sawaya R: Metastasis of esophageal carcinoma to the brain. Cancer 98: 1925-1933, 2003.

6. Yamamoto T, Kuroda J, Takezaki T, Shinojima N, Hide T, Makino K, Nakamura H, Yano S, Nishi T and Kuratsu J: Characteristics of brain metastases from esophageal carcinoma. Surg Neurol Int 5: 137, 2014.

7. Takeshima H, Kuratsu J, Nishi T, Soyama N, Miura M, Masumitsu T and Ushio Y: Metastatic brain tumours from oesophageal carcinoma: neuro-imaging and clinicopathological characteristics in Japanese patients. Acta Neurochir (Wien) 143: 31-35, discussion 35-36, 2001.

8. Gabrielsen TO, Eldevik OP, Orringer MB and Marshall BL: Esophageal carcinoma metastatic to the brain: clinical value and cost-effectiveness of routine enhanced head CT before esophagectomy. AJNR Am J Neuroradiol 16: 1915-1921, 1995.

9. Lutterbach J, Cyron D, Henne K and Ostertag CB: Radiosurgery followed by planned observation in patients with one to three brain metastases. Neurosurgery 52: 1066-1073, discussion 1073-1074, 2003.

10. Kanemoto A, Hashimoto T, Harada H, Asakura H, Ogawa H, Furutani K, Boku N, Nakasu Y and Nishimura T: Occurrence and clinical features of brain metastasis after chemoradiotherapy for esophageal carcinoma. J Radiat Res (Tokyo) 52: 509-515, 2011.

11. Harders SW, Madsen HH, Hjorthaug K, Arveschoug AK, Rasmussen TR, Meldgaard P, Hoejbjerg JA, Pilegaard HK, Hager H, Rehling $M$ and Rasmussen F: Mediastinal staging in non-small-cell lung carcinoma: computed tomography versus F-18-fluorodeoxyglucose positron-emission tomography and computed tomography. Cancer Imaging 14: 23, 2014.

12. Seute T, Leffers P, ten Velde GP and Twijnstra A: Detection of brain metastases from small cell lung cancer: consequences of changing imaging techniques (CT versus MRI). Cancer 112: $1827-1834,2008$

13. Hjorthaug K, Højbjerg JA, Knap MM, Tietze A, Haraldsen A, Zacho HD, Kramer SM and Borghammer P: Accuracy of 18F-FDG PET-CT in triaging lung cancer patients with suspected brain metastases for MRI. Nucl Med Commun 36: 1084-1090, 2015.

14. Yoshida S: Brain metastasis in patients with esophageal carcinoma. Surg Neurol 67: 288-290, 2007.

15. Withers HR and Lee SP: Modeling growth kinetics and statistical distribution of oligometastases. Semin Radiat Oncol 16: 111-119, 2006.

16. Corbin KS, Hellman S and Weichselbaum RR: Extracranial oligometastases: a subset of metastases curable with stereotactic radiotherapy. J Clin Oncol 31: 1384-1390, 2013.

17. Rastogi S, Gulia S, Bajpai J, Ghosh J and Gupta S: Oligometastatic breast cancer: a mini review. Indian J Med Paediatr Oncol 35: 203-206, 2014. 